\title{
Instructing human macrophage polarization by stiffness and glycosaminoglycan functionalization in 3D collagen networks
}

\author{
Markus Friedemann ${ }^{1}$, Liv Kalbitzer ${ }^{1}$, Sandra Franz ${ }^{2}$, Stephanie Moeller, Matthias Schnabelrauch ${ }^{3}$, Jan- \\ Christoph Simon'2, Tilo Pompe ${ }^{1}$ and Katja Franke ${ }^{1^{*}}$ \\ ${ }^{1}$ Institute of Biochemistry, Universität Leipzig, 04103 Leipzig, Germany \\ ${ }^{2}$ Department of Dermatology, Venerology and Allergology, Universitätsklinikum Leipzig, 04103 Leipzig, Germany \\ ${ }^{3}$ INNOVENT e. V., Biomaterials Department, 07745 Jena, Germany \\ * corresponding author \\ katja.franke@uni-leipzig.de
}

Keywords: human macrophages, extracellular matrix, collagen, glycosaminoglycan, stiffness

\begin{abstract}
Dynamic alterations of composition and mechanics of the extracellular matrix (ECM) are suggested to modulate cellular behavior including plasticity of macrophages (MPhs) during wound healing. In this study, engineered 3D fibrillar matrices based on naturally occurring biopolymers (collagen I, glycosaminoglycans (GAGs)) were used to mimic matrix stiffening as well as modification by sulfated and non-sulfated GAGs at different stages of wound healing. Human MPhs were found to sensitively respond to these microenvironmental cues in terms of polarization towards pro-inflammatory or wound healing phenotypes over 6 days in vitro. MPhs exhibited a wound healing phenotype in stiffer matrices as determined by protein and gene expression of relevant cytokines (IL10, IL12, TNF $\alpha$ ). Presence of sulfated and non-sulfated GAGs inhibited this polarization effect. Furthermore, control experiments on 2D matrices stressed the relevance of using stiffness-controlled 3D matrices, as MPhs showed a reciprocal polarization behavior depending on GAG presence. Hence, the results indicate a strong influence of dimensionality, stiffness, and GAG presence of the biomaterial scaffold on MPh polarization and emphasize the need for matrices closely mimicking the 3D in vivo context with a variable stiffness and GAG composition in in vitro studies.
\end{abstract}

\section{Introduction}

Biochemical and biophysical properties of the extracellular matrix (ECM) have already been shown to influence cellular behavior in pathological and physiological processes of many tissues. ${ }^{[1,2]}$ The 3D microstructure and stiffness of the ECM as well as presence of sulfated and non-sulfated glycosaminoglycans (GAGs) are specifically discussed as regulating microenvironmental cues. ${ }^{[3,4]}$ 3D ECM microstructure and stiffness are known to control adhesion receptor activation and assembly as well as downstream cellular contractility, proliferation and differentiation. ${ }^{[5-9]}$ Furthermore, GAGs can bind growth factors and cytokines, and present them to nearby cells in an activated or inactivated state. Due to the GAG-cytokine interaction, GAGs control local cytokine storage and release that modulates cellular chemotaxis and paracrine signaling. ${ }^{[10,11]}$

Important regulatory functions of the ECM have been described for different stages of wound healing. ${ }^{[12]}$ In wound healing the ECM is characterized by very dynamic changes in terms of composition, microstructure and mechanics e.g. the remodeling of fibrin-rich initial ECM by proteinases and fibrillar collagen deposition. ${ }^{[13]}$ Moreover, the profiles of inflammatory and anti-inflammatory cytokines change in the time course of progressing wound healing, a process regulated by immune cells. ${ }^{[14]}$ In this process macrophages (MPhs) are known to play a central role. Secretion of cytokines for paracrine stimulation of other cell types, matrix resolution by matrix-metalloproteinases or moderation of scar formation during wound repair are some of the processes facilitated by MPhs. ${ }^{[14-16]}$ These various functions of MPhs are highly dynamic given the strong plasticity of MPh phenotype, which is suggested to be dependent on the interplay with surrounding cells and the ECM microenvironment. ${ }^{[17,18]}$

Roughly, the MPh phenotypes can be classified into pro-inflammatory ' $\mathrm{M} 1$ ' MPhs and alternatively activated wound healing 'M2' MPhs. ${ }^{[14,19,20]}$ 'M1' MPhs are characteristic for the early inflammatory phase of wound healing with a production of high levels of cytokines like interleukin (IL) 1, 6, 8, 12 and tumor necrosis factor $\alpha$ (TNF $\alpha$ ). 'M2' MPhs are related to a later stage of wound healing, so-called proliferation phase of fibroblasts and other tissue cells, with the expression of high levels of antiinflammatory IL10 and pro-repair mediators resistinlike molecule alpha (Relm- $\alpha$, and tissue growth factor (TGF $\beta$ ). The simplified classification in ' $\mathrm{M} 1$ ' and ' $\mathrm{M} 2$ ' 
MPhs is known to exhibit smooth transitions into each other enabling the control of the healing process. ${ }^{[19,20]}$

Besides the detailed knowledge on single molecular regulators of MPh function, a full understanding of combined molecular mechanisms and their interplay with the surrounding ECM is not available. In order to better support progress in challenging therapeutic situations like chronic wounds, in-depth in vitro studies in biomimetic ECM microenvironments are suggested to elucidate MPh behavior at such multi-parameter conditions. Engineered ECM mimics allow to address in a controlled manner the impact of specific microenvironmental cues like stiffness changes during ECM remodeling or presence of different GAGs to mediate paracrine function of inflammatory or anti-inflammatory cytokines. ${ }^{[17]}$

Some biomaterial approaches already tried to address the impact of ECM properties on MPh polarization. A collagen type I (Coll) surface functionalized with non-covalently bound synthetically sulfated hyaluronan (HA) was shown to induce a wound healing phenotype in human monocyte derived MPhs. ${ }^{[21]}$ Enhanced stiffness of a PEG based hydrogel support led to a cytokine profile typical for inflammatory MPh in vitro after stimulation with LPS or to severe foreign body reaction in mice. ${ }^{[22]}$ Furthermore, substrate morphology was described to exert a strong influence on MPh phenotypes . 23] However, either the materials used have been 2D surfaces missing in vivolike properties of a 3D porous biomaterial or the 3D materials applied were not sufficiently characterized with respect to composition, mechanics and microstructure. In this respect, 3D fibrillar matrices from naturally derived biopolymers like decellularized matrices or Coll based networks have an outstanding function, as they are able to reconstitute the nano- and microstructure of the in vivo ECM. ${ }^{[24-27]}$ Using advanced engineering and analysis tools, Coll based matrices can be constructed with defined topological, mechanical and compositional properties. ${ }^{[28-35]}$

In this study, we utilized these tools and designed 3D Coll based matrices with defined stiffness and presence of sulfated and non-sulfated GAGs to investigate their modulating impact on the phenotype of human MPhs. The variation of these ECM parameters addresses the dynamic variation of ECM characteristics during wound healing. We used granulocyte-macrophage colony-stimulating factor (GM-CSF) to induce differentiation of human monocytes into MPhs with an inflammatory phenotype and examined phenotypic changes by analyzing levels of anti-inflammatory (IL10) and inflammatory (IL12 and TNF $\alpha$ ) cytokines under the influence of the biomimetic 3D ECM. Control experiments on similar 2D ECM were performed to highlight the importance of using in vivo-like 3D ECM matrices with tunable stiffness and GAG composition for characterizing MPh polarization in a 3D context.

\section{Materials and Methods}

2.1. Synthesis of Molecular Weight-Defined NonSulfated and Sulfated Hyaluronan A partly degraded, non-sulfated hyaluronan (HA) and a high-sulfated hyaluronan (sHA with a degree of sulfation (D.S.) of about 3 (D.S. = average number of sulfate groups per disaccharide repeating unit) with comparable molecular weights (MW) of about $57 \mathrm{kDa}$ were used in this study. HA was prepared by ozonolysis of high MW, native HA (from Streptococcus, MW 1.1 MDa, Aqua Biochem, Dessau, Germany), as described before. ${ }^{[29,36]}$ Briefly, an $1 \%$ aqueous solution of high MW HA was treated with ozone, prepared with an ozone generator COM-AD-02 (ANSEROS Klaus Nonnenmacher, Tübingen, Germany) for $2 \mathrm{~h}$. The ozone concentration amounted to approx. $60 \mathrm{~g} \mathrm{~m}^{-3}$ and a flow rate of 20 to $30 \mathrm{I} \mathrm{h}^{-1}$ was used. Finally, $\mathrm{N}_{2}$ was passed through the solution for $30 \mathrm{~min}$ to expel free ozone. The remaining clear solution was dialyzed against distilled water, lyophilized and dried under vacuum. The HA was obtained with 75 to $85 \%$ yield. sHA derivatives were synthesized and characterized as described previously. ${ }^{[37]}$

\subsection{Reconstruction and GAG Functionalization of $2 D$} and 3D Collagen Networks Fibril formation of rat tail collagen type I (Coll, Corning, New York, USA) $\left(2.5 \mathrm{mg} \mathrm{ml}^{-1}\right)$ in phosphate buffer ( $\left.\mathrm{pH} 7.5\right)$ was performed at $37{ }^{\circ} \mathrm{C}$ and $95 \%$ humidity for 60 min as described previously. ${ }^{[28]}$ Prepared 3D Coll networks have a thickness of about $300 \mu \mathrm{m}$ and a mean pore size and fibril diameter of $9 \mu \mathrm{m}$ and $700 \mathrm{~nm}$, respectively, as routinely characterized. (For details on methodoloy see ${ }^{[28,38]}$.) For improved handling, the fibrillar networks were covalently immobilized on poly(styrene-alt-maleic anhydride) copolymer coated glass coverslips as described elsewhere. ${ }^{[28,39]} \mathrm{HA}$ and sHA modification of Coll networks were performed as previously reported. ${ }^{[29]}$ Briefly, Coll I networks were incubated with $\mathrm{HA}$ or SHA $\left(0.2 \mathrm{mg} \mathrm{ml}^{-1}\right)$ in 2-(Nmorpholino)ethanesulfonic acid (MES, $0.1 \mathrm{M}, \mathrm{pH}$ 5, Sigma Aldrich, Munich, Germany) for $2 \mathrm{~h}$ at room temperature. Subsequently, covalent $H A$ or $s H A$ binding was induced by incubation for $2 \mathrm{~h}$ at room temperature in $\mathrm{N}$-(3-Dimethylaminopropyl)- $\mathrm{N}^{\prime}$ ethylcarbodiimide hydrochloride (20 mM) (EDC, Sigma Aldrich) in MES (0.1 M, pH 5). Crosslinking of Coll networks was performed in the same way, but, without prior incubation with $\mathrm{HA} / \mathrm{sHA}$ solutions. Finally, Coll networks were washed 3 times in 
phosphate buffered saline (PBS, Biochrom, Berlin, Germany) and incubated for equilibration overnight in cell culture medium before usage. 2D surfaces were prepared from 3D networks. The networks were rinsed and collapsed by dehydration overnight.

\subsection{Stiffness Measurements of Collagen Networks} For micromechanical characterization of networks, an effective Young's modulus, $E$, was determined by fitting the retract part of the force-distance curves recorded by colloidal probe force spectroscopy using the Hertz model, as described previously. ${ }^{[38]}$ Measurements were performed using a Nanowizard III (JPK Instruments, Berlin, Germany) and probes with a manually attached $50 \mu \mathrm{m}$ glass microbead (Polyscience Europe GmbH, Eppelheim, Germany) to a tipless HQ-CSC38 cantilever (NanoAndMore, Germany) with a spring constant of approx. $0.1 \mathrm{~N} \mathrm{~m}$ ${ }^{1}$. The exact spring constant was determined by the thermal noise method. ${ }^{[40]}$ All samples were characterized in PBS at room temperature. A minimum of 27 force-distance curves at 3 positions per sample in 3 independent experiments was measured.

\subsection{Human Monocyte Isolation and Macrophage} Differentiation Heparinized blood of human healthy volunteers after informed signed consent was purchased from the Institute of Transfusion Medicine at Universität Leipzig. Monocytes were enriched by density-gradient centrifugation (Ficoll-Paque PLUS, GE Healthcare, Freiburg, Germany) and isolated using CD14 negative depletion (Miltenyi Biotec, Bergisch Gladbach, Germany). Purity of CD14 positive cells was assessed by flow cytometry (FACS Vantage SE TurboSort, BD, Heidelberg, Germany) at about 95\% CD14 positive cells. $6 \cdot 10^{4}$ monocytes in RPMI $(500 \mu \mathrm{l}$, Biochrom) were cultivated per network (Coll, EDC, HA, sHA) or bare well (TCP; 24-well plate; Greiner BioOne, Frickenhausen, Germany). RPMI was supplemented with heat inactivated FCS (10\%) (Biochrom) and granulocyte macrophage colony stimulating factor (GM-CSF, $20 \mathrm{ng} \mathrm{ml}^{-1}$ ) or macrophage colony stimulating factor (MCSF, $50 \mathrm{ng} \mathrm{ml}^{-1}$ ) both from R\&D, for monocyte to inflammatory or anti-inflammatory MPh differentiation. Cell culture was performed at $37^{\circ} \mathrm{C}$, $5 \% \mathrm{CO}_{2}, 95 \%$ humidity for 6 days. Medium was renewed every $3^{\text {rd }}$ day. At day 6 medium was additionally supplemented with LPS (Sigma Aldrich, $100 \mathrm{ng} \mathrm{ml}^{-1}$ ) to induce cytokine secretion for $24 \mathrm{~h}$ or $4 \mathrm{~h}$ for gene expression analysis. For a visualization of the experimental set up see Figure 1.

2.5. Analysis of Secreted and Intracellular Cytokines and GM-CSF Solution Depletion Monocyte derived MPhs were characterized with respect to their inflammatory and anti-inflammatory polarization after cultivation in GM-CSF or MCSF supplemented medium on bare cell culture plastic (TCP) by cytokine ELISA. To analyze the influence of differently modified Coll networks (Coll, EDC, HA, sHA) on MPh polarization in presence of GM-CSF (inflammatory condition), secreted and intracellular cytokines were assessed by cytokine ELISA of cell culture supernatants and by confocal laser scanning microscopy. Solution depletion of GM-CSF was measured after incubation of cell culture medium for $24 \mathrm{~h}$ with the different Coll networks using ELISA. ELISA detecting IL10, TNF $\alpha$, GM-CSF (eBioscience, Frankfurt am Main, Germany) and IL12(p40) (BD Bioscience) were performed according to the manufacturer's instructions. Released cytokine concentrations were analyzed in cell culture supernatants after MPh stimulation with LPS $\left(100 \mathrm{ng} \mathrm{ml}^{-1}\right)$ for $24 \mathrm{~h}$. As proliferation of MPhs is known to be negligible, results were normalized to initial cell number. (The fact of negligible proliferation was proven by means of WST-1 assay (Roche, Penzberg, Germany) in pre-experiments.) The comparable cell number of different networks is also supported by similar RNA levels observed in the gene expression experiments. Supernatants were stored at $-20^{\circ} \mathrm{C}$ prior measurement.

For intracellular IL10 and IL12 levels differentiated and LPS stimulated (6 days $+24 \mathrm{~h}$, respectively) MPhs were washed, fixed and permeabilized at day 7 of cultivation inside the different Coll networks with PBS containing $\mathrm{Ca}^{2+}$ and $\mathrm{Mg}^{2+}$ (Biochrom) at $37^{\circ} \mathrm{C} 3$ times, paraformaldehyde (4\%, Roth, Karlsruhe, Germany) for $15 \mathrm{~min}$ at $37^{\circ} \mathrm{C}$ and Triton X100 (0.1\%, Roth, Karlsruhe, Germany) for $15 \mathrm{~min}$ at room temperature, respectively. Afterwards, the fixed samples were rinsed 3 times with PBS and subsequently incubated for $1 \mathrm{~h}$ in blocking solution containing bovine serum albumin (5\%) (BSA; Sigma Aldrich) in PBS to minimize nonspecific antibody binding. Monoclonal rat anti human IL10 (JES3-9D7, eBioscience) conjugated with Alexa488, polyclonal rabbit anti human IL12 (Bioss, Woburn, USA) conjugated with Alexa555 and DAPI (Sigma Aldrich) were incubated for $1 \mathrm{~h}$. Samples were rinsed using blocking solution 3 times and confocal stacks at an xy-size of $512 \times 512$ pixels $(160 \times 160 \mu \mathrm{m})$ and a $5 \mu \mathrm{m}$ image distance were taken using a confocal laser scanning microscope (LSM700) and a 40x water immersion objective (both Carl Zeiss Microscopy, Jena, Germany). Mean fluorescence intensity of intracellular cytokines was analyzed from z-projections of confocal stacks in relation to cell number revealed by DAPI. 20 cells per sample were assessed at minimum.

2.6. IL10 Gene Expression Analysis For quantitative real-time polymerase chain reaction (RT-qPCR) analysis, GM-MPh inside the different Coll networks 


\section{Isolation} Differentiation

Analysis

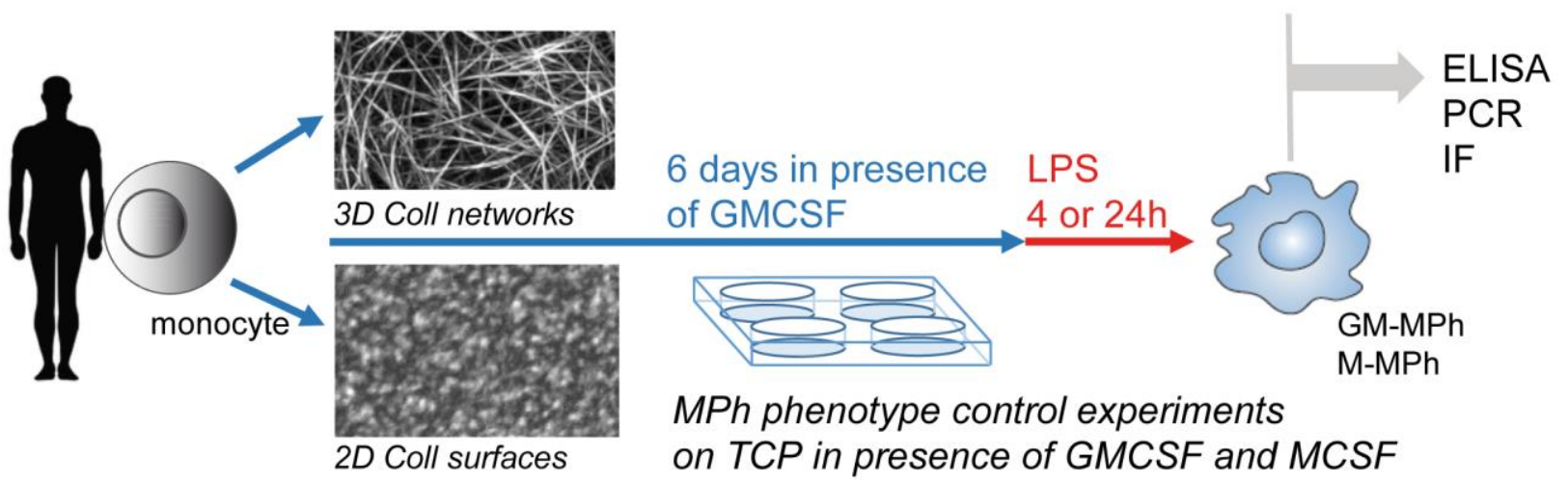

Figure 1. Experimental setup. Freshly isolated monocytes were cultivated either inside 3D Coll networks or on top of 2D Coll matrices in presence of GM-CSF to investigate MPh polarization. To demonstrate the generation of an inflammatory and a wound healing MPh phenotype in the presence of GM-CSF or MCSF and the expression of cytokines IL10, IL12 and TNF $\alpha$ as phenotypic markers, control experiments were performed in tissue culture plastic (TCP). LPS stimulation was performed during 4 or 24 h prior to analysis of cytokine profile via PCR, ELISA or immunofluorescence (IF).

were LPS stimulated for $4 \mathrm{~h}$ at day 6 . RNA was isolated using TRIzol ${ }^{\circ}$ Reagent (Life Technologies, Carlsbad, USA) and ReliaPrep ${ }^{\text {TM }}$ RNA Tissue Miniprep (Promega, Mannheim, Germany). The RNA amount was determined by spectrophotometry using a $\mu$-Cuvette (Eppendorf, Wesseling-Berzdorf, Germany). The synthesis of cDNA was performed according suppliers protocol $\quad$ GoScript $^{\text {TM }}$ Reverse Transcription, Promega). RT-qPCR was performed with a Thermocycler CFX ConnectTM Real-Time PCR Detection System (Bio-rad, München, Germany) using GoTaq ${ }^{\circledR}$ qPCR Master Mix (Promega). The following primers were applied: IL10 (fw CCCAAGCTGAGAACCAAGAC; rW AAGGCATTCTTCACC TGCTC), GNB2L (fW GAGTGTGGCCTTCTCCTCTG; rW GCTTGCAGTTAGCCAGGTTC) and MRTO4 (fW AAAGC AGCTTTCACTGTGAGC; rw CAGAGTCACCACACCTCTC TTG) (all from eurofins genomics, Ebersberg, Germany). The PCR procedure was set as follows: denaturation for $5 \mathrm{~min}$ at $95^{\circ} \mathrm{C} ; 45$ amplifications of denaturation $\left(15 \mathrm{~s}\right.$ at $\left.95^{\circ} \mathrm{C}\right)$, annealing under primerspecific conditions $(20 \mathrm{~s})$ and target gene-specific extension $\left(20 \mathrm{~s}\right.$ at $\left.72^{\circ} \mathrm{C}\right)$ with subsequent fluorescence measurement. Melting curves were recorded for control of the specificity of the PCR products. IL10 gene expression was normalized to the non-regulated reference genes GNB2L and MRTO4. Results are expressed as fold induction with respect to GM-MPh without LPS stimulation.

2.7. Cell Invasion Analysis Cell invasion was analyzed at day 7 after $24 \mathrm{~h}$ LPS stimulation. After fixation and permeabilization as described above, cells were stained with DAPI (Invitrogen, Germany) for $30 \mathrm{~min}$ at room temperature and subsequently rinsed 3 times with PBS. Image stacks in vertical direction were taken using an AxioObserver.Z1 with scanning stage and a 10x objective (Carl Zeiss Microscopy). Image resolution, size and $z$-distance were $692 \times 520$ pixels, $886 \times 666 \mu \mathrm{m}$ and $5 \mu \mathrm{m}$. Cell localization was analyzed using DAPI signal, cells $>50 \mu \mathrm{m}$ below Coll network surface were defined as invasive cells.

2.8. Data Analysis and Statistics Experiments were performed at least in seven independent replicates, if not otherwise stated. Error bars indicate standard error of the mean. Levels of statistical significance were determined using a paired Wilcoxon signedrank test in OriginPro 8 (OriginLab Corp., USA. Significance level was set at 0.05 .

\section{Results and Discussion}

\subsection{Experimental Setup}

An elongated inflammatory phase was discussed as the critical issue in chronic wounds. Therefore, we focused in our study on the capabilities of the different Coll networks to modulate the inflammatory phenotype of MPhs by the properties of the ECM environment. Differentiation of inflammatory MPhs (GM-MPh) was induced by GM-CSF, which has been described to generate MPhs similar to those found at inflammation-site. ${ }^{[41]}$ Figure 1 shows our experimental setup. Freshly isolated human monocytes were directly placed in contact with the Coll networks and differentiated into GM-MPhs over 7 days including a final LPS stimulation ( $4 \mathrm{~h}$ for PCR analysis or $24 \mathrm{~h}$ for ELISA and immunofluorescence) to induce their activation. We characterized the polarization status of MPhs towards inflammatory or wound healing phenotypes analyzing expression and release of the cytokines IL10, IL12 and TNF $\alpha$, which is in accordance with the recently stated recommendations for MPh phenotype characterization. ${ }^{[41]}$ To verify the suitability of GM- 

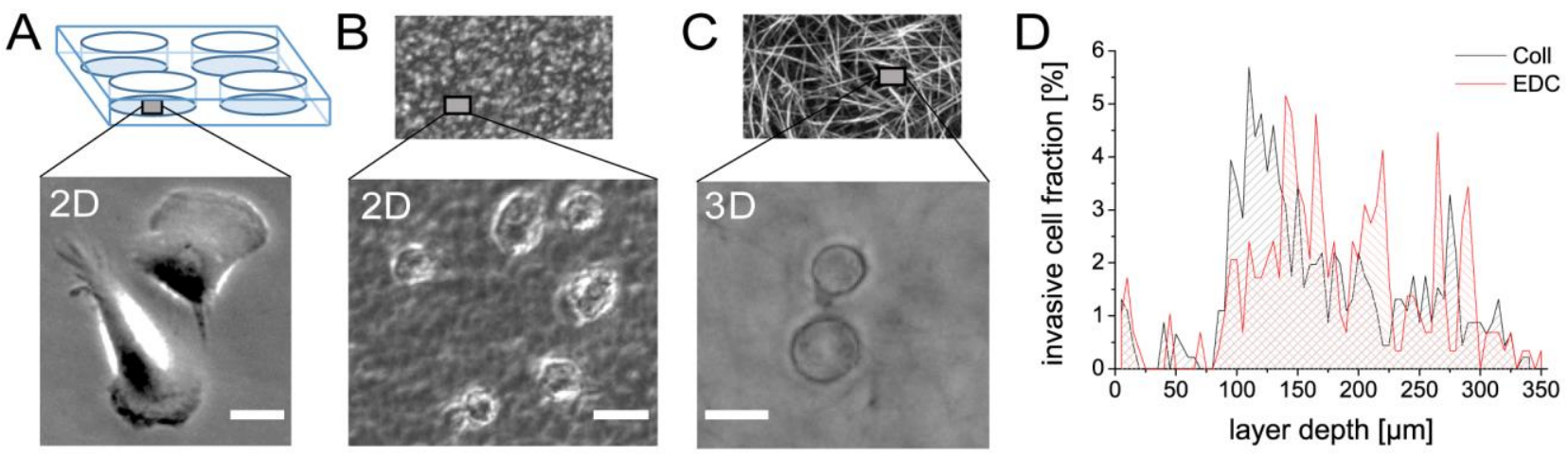

Figure 2. Morphology and invasion of GM-MPh. (A-C) Representative microscopy images show the strong difference in cell morphology in dependence on cell culture substrates being tissue culture plastics (TCP) (A), dense 2D Coll matrices (B), and 3D Coll networks (C). (Scale bar: $20 \mu \mathrm{m})(D)$ Cell invasion analysis of GM-MPh was performed by microscopy analysis of DAPI-stained cell nuclei. GM-MPhs were equally distributed within the Coll and EDC network layer after 7 days. Cell distribution inside HA and sHA samples showed a similar distribution as EDC. The histogram shows the analysis of one representative experiment out of 4 experiments.

CSF to generate an inflammatory 'M1' (GM-MPh) and the usage of cytokines IL10, IL12 and TNF $\alpha$ as relevant phenotypic markers, initial control experiments were performed on tissue culture plastics before the start of our study. The need for more in vivo-like 3D environment was additionally verified by control experiments in $2 \mathrm{D}$ settings of collapsed Coll networks.

Freshly isolated monocytes (in presence of GMCSF) were cultured in 3D fibrillar networks based on Coll that were EDC crosslinked or functionalized with $\mathrm{HA}$ or sHA to investigate the impact of the GAG presence (sulfated or non-sulfated) as well as of matrix stiffness of the cellular microenvironment on the resulting MPh phenotype. EDC crosslinking increases the stiffness of Coll networks and can additionally be used to covalently attach $\mathrm{HA}$ and sHA to the fibrillar Coll networks, while leaving network topology (pore size $(9 \pm 1.2 \mu \mathrm{m})$, fibril diameter $700 \pm 18 \mathrm{~nm}$ ) unaffected as described previously. ${ }^{[2]}$ Thus, such 3D Coll based matrices can partly mimic the in vivo-like fibrillar microstructure similar to dermis and connective tissue, in particular concerning the porous microstructure of the distinct Coll fibrils. ${ }^{[1,42]}$ Depending on the different network modification, the set of engineered networks used in the following cell experiments is referred to as: Coll pure collagen network; EDC - EDC crosslinked Coll network; HA - Coll network covalently functionalized with HA using EDC; sHA - Coll network covalently functionalized with sHA using EDC. Besides the different GAG functionalization, the stiffness of these networks was verified to follow the previously reported trends of Young's modulus, indicating a significant stiffness increase for EDC, HA and sHA networks in comparison to pure Coll (Coll 27.1 $\pm 9.8 \mathrm{~Pa}, \mathrm{EDC} 57.5 \pm 25.9 \mathrm{~Pa}, \mathrm{HA} 73.6 \pm 27.5 \mathrm{~Pa}$ and sHA $118.5 \pm 34 \mathrm{~Pa}$, for details see Supplemental Figure S1).
MPh phenotypes were defined via expression of inflammatory and anti-inflammatory cytokines during the experiments. As reference we used standard differentiation protocols (using growth factors GMCSF versus MCSF) with subsequent LPS stimulation to receive inflammatory (M1, GM-MPh) or wound healing (M2, M-MPh) MPh phenotypes. ${ }^{[43]}$ Figure S2A shows the reference experiments on standard tissue culture plastics for GM-MPh and M-MPh differentiation and the expression of cytokines IL10, IL12, TNF $\alpha$. The inflammatory phenotype of GM-MPh was proved by an enhanced secretion of inflammatory cytokines IL12 and TNF $\alpha$ and a decreased secretion of anti-inflammatory IL10. ${ }^{[41]}$ In contrast, the anti-inflammatory phenotype of M-MPh was reflected by an enhanced secretion of antiinflammatory IL10 and lower levels of inflammatory IL12 and TNF $\alpha .{ }^{[41]}$ By calculating the ratio of IL10 to IL12 of the two different phenotypes we demonstrate herein the usage of this ratio as an useful tool to differentiate between anti-inflammatory and inflammatory MPh phenotypes, as already suggested by Franz et al. ${ }^{[21]} \mathrm{M}-\mathrm{MPh}$ showed a 24.5 fold higher ratio compared to GM-MPh (Figure S2B).

\subsection{Cell Morphology and Cell Invasion in 2D and 3D Culture Conditions}

At first we assessed cell morphology of 2D versus 3D cell culture conditions as this is known to be a straightforward indicator of mechanosensitivity of cells, and also to effect downstream cell signaling. ${ }^{[4]}$ Furthermore, invasion of cells in 3D matrices was determined to ensure the proper 3D context in cell studies. Non-coated tissue culture plastics (TCP) and collapsed and dense 2D Coll matrices acted as controls in comparison to 3D Coll networks. We revealed enormous differences in cell morphology with a mainly flattened, spread cell shape of about 40 to $50 \mu \mathrm{m}$ in diameter on TCP and a mainly round cell 
shape on $2 \mathrm{D}$ and in $3 \mathrm{D}$ Coll matrices with cell diameters of about 20 to $30 \mu \mathrm{m}$ (Figure $2 \mathrm{~A}-\mathrm{C}$ ). These results already indicate the importance to use in vivolike ECM composition for in vitro cell culture experiments.

The analysis of GM-MPh invasion into the 3D networks after 7 days revealed well distributed cells inside the Coll networks with minor difference between Coll and EDC crosslinked networks (Figure 2D). Cell distribution inside HA and sHA networks was similar to EDC networks. This equal distribution of GM-MPh within our 3D Coll based networks is an important requirement allowing the subsequent studies on the impact of matrix stiffness and GAG modification on MPh polarization in the 3D context.

\subsection{Cytokine Secretion in 2D versus 3D Culture Conditions and GM-CSF Solution Availability}

The depletion of cytokines and growth factors from cell culture medium through their binding to GAGcontaining ECM networks is well-described in several in vitro and in vivo studies. ${ }^{[45]}$ Therefore, we first evaluated the GM-CSF concentration in cell culture medium on the different networks to verify an equal availability of GM-CSF during the period of MPh differentiation. These pre-experiments revealed no differences in available GM-CSF concentrations between the different matrix conditions with detected GM-CSF concentration of about $6.8 \pm$ $0.3 \mathrm{ng} \mathrm{ml}^{-1}$ in cell culture medium after 1 day. This lower concentration in comparison to that initially applied (20 $\left.\mathrm{ng} \mathrm{ml}^{-1}\right)$ may result from degradation effects in the medium due to the reduced stability of GM-CSF as shown for other growth factors. ${ }^{[46]}$ Importantly, in all experimental settings comparable GM-CSF concentrations were present for MPh differentiation.

Next, the influence of the different 2D and 3D matrix conditions on MPh polarization was investigated by means of cytokine secretion of IL10, IL12 and TNF $\alpha$. Cytokine levels were evaluated after 6 days of monocyte differentiation in GM-CSF supplemented medium with an additional LPSstimulation for the final $24 \mathrm{~h}$ of culture.

The cytokine profile on 3D networks showed a significantly higher secretion of the pro-resolution

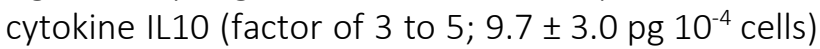
for GM-MPh inside EDC crosslinked networks in comparison to all other 3D matrix conditions, while the secretion of IL12 and TNF $\alpha$ - both typical markers for an inflammatory MPh phenotype - were by trend similar or reduced in these networks (Figure $3 \mathrm{~A}$ ). Importantly, GM-MPh on 2D matrices showed a different behavior. GM-MPh on 2D Coll surfaces secreted much higher amounts per cell of all three cytokines (IL10, IL12, TNF $\alpha$ ) in comparison to 3D culture conditions (roughly factor 10). Furthermore, on 2D HA and SHA surfaces cytokine release was higher (IL10) or lower (IL12, TNF $\alpha$ ) compared to the corresponding 3D matrices.

As the usage of primary human donor cells often results in a strong variance of total amounts of secreted cytokines, the ratio of IL10 to IL12 is a useful tool to differentiate between MPh phenotypes. ${ }^{[21]}$ We calculated this ratio relative to Coll as depicted in
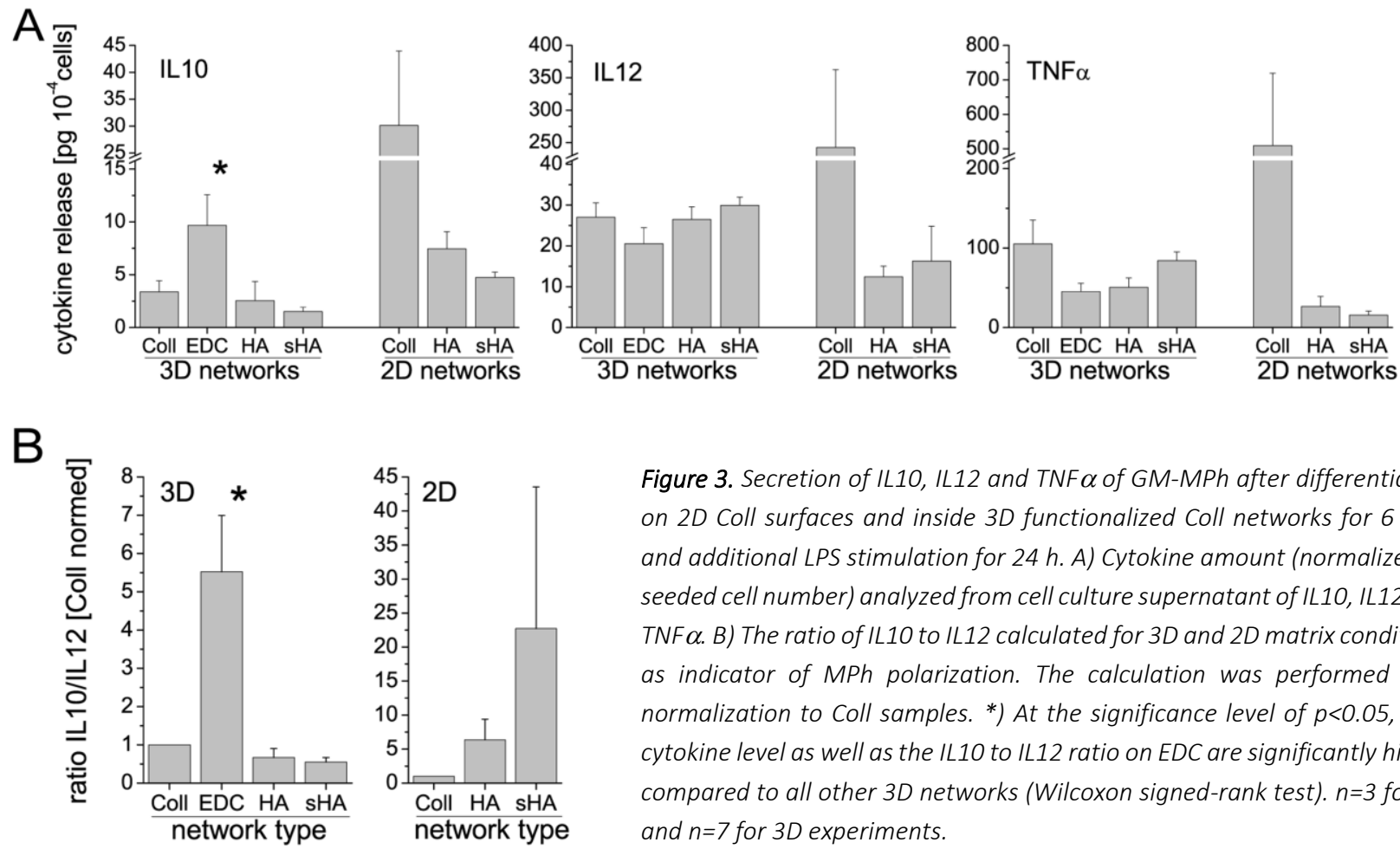

Figure 3. Secretion of IL10, IL12 and TNF $\alpha$ of GM-MPh after differentiation on 2D Coll surfaces and inside 3D functionalized Coll networks for 6 days and additional LPS stimulation for $24 \mathrm{~h}$. A) Cytokine amount (normalized by seeded cell number) analyzed from cell culture supernatant of $I L 10, I L 12$ and TNF $\alpha$. B) The ratio of IL10 to IL12 calculated for 3D and 2D matrix conditions as indicator of MPh polarization. The calculation was performed with normalization to Coll samples. *) At the significance level of $p<0.05,1 L 10$ cytokine level as well as the IL10 to IL12 ratio on EDC are significantly higher compared to all other $3 D$ networks (Wilcoxon signed-rank test). $n=3$ for $2 D$ and $n=7$ for $3 D$ experiments. 
Figure 3B. For 3D conditions IL10/IL12 ratio is significantly increased for the EDC networks in comparison to Coll (by a factor of 5), while it remains on similar levels for the HA and sHA networks. Obviously, no simple correlation of network stiffness and cytokine expression could be found as HA and sHA networks have similar or somewhat increased stiffness levels as compared to EDC crosslinked networks. As for the cytokine profile, the IL10/IL12 ratio shows a different trend for $2 \mathrm{D}$ matrices with an increasing ratio for HA and sHA compared to Coll.

These results suggest that MPhs are instructed to polarize toward an anti-inflammatory phenotype (low TNF $\alpha, I L-12$ and high IL-10 ${ }^{[41]}$ ) by stiffer 3D matrices even under inflammatory conditions (GMCSF and LPS). The presence of both GAGs, nonsulfated and sulfated HA, inhibited this stiffnessmediated effect on MPh polarization. The results not only reveal the importance of matrix stiffness and the presence of GAG for MPh polarization studies. They also demonstrate the impact of the $3 \mathrm{D}$ environment by the comparative analysis of the effect of the 3D networks with corresponding 2D control matrices (collapsed Coll networks with optional GAG modification). MPh on the 2D matrices showed a different behavior with a trend toward an antiinflammatory phenotype in the presence of the GAGs. Importantly, these findings are consistent with previous reports demonstrating other types of 2D Coll matrices with adsorbed sHA to modulate the differentiation of human inflammatory MPhs towards an alternative polarization profile. ${ }^{[21]}$

As we observed a different polarization profile in $3 \mathrm{D}$ matrices in comparison to the $2 \mathrm{D}$ controls, depletion effects through the binding of secreted cytokines inside the 3D matrices was considered a possible influence on measured cytokine concentrations in the supernatants. While direct quantification of small amounts of bound cytokines in Coll networks within a cell culture context is currently hard to achieve because of the strong protein background, we decided to follow an indirect approach. We verified cytokine expression profiles of GM-MPh by determination of intracellular levels of IL10 and IL12 as well as gene expression analysis of IL10 (Figure 4A,B). The immunofluorescence analysis of intracellular IL10 and IL12 of GM-MPh inside the 3D matrices by confocal laser scanning microscopy showed the same MPh polarization profile as found for the cytokine release analysis. The IL10/IL12 ratio was significantly increased in EDC crosslinked matrices in comparison to non-modified Coll, while an inhibition of this anti-inflammatory polarization was found for the GAG modified matrices. Therefore, we concluded that the evaluation of the cytokine response and the IL10/IL12 secretion profile is not influenced by cytokine binding effects of the investigated 3D fibrillar Coll matrices. This is further supported by IL10 gene expression analysis showing a significant upregulation of IL10 expression in MPh differentiated in the EDC crosslinked matrices in comparison to all other matrices (Figure 4C).

Taken together, our data on cytokine induction including cytokine release, intracellular protein levels and cytokine gene expression clearly demonstrate a polarization of GM-MPh towards an antiinflammatory phenotype in 3D EDC crosslinked matrices. Although we can exclude that the investigated cytokines are depleted in the culture through steady binding to the $3 \mathrm{D}$ Coll matrices additive effects on autocrine signaling events via short-term binding of these or other factors produced from the MPh to the 3D matrices regulating their presentation to the cells cannot be
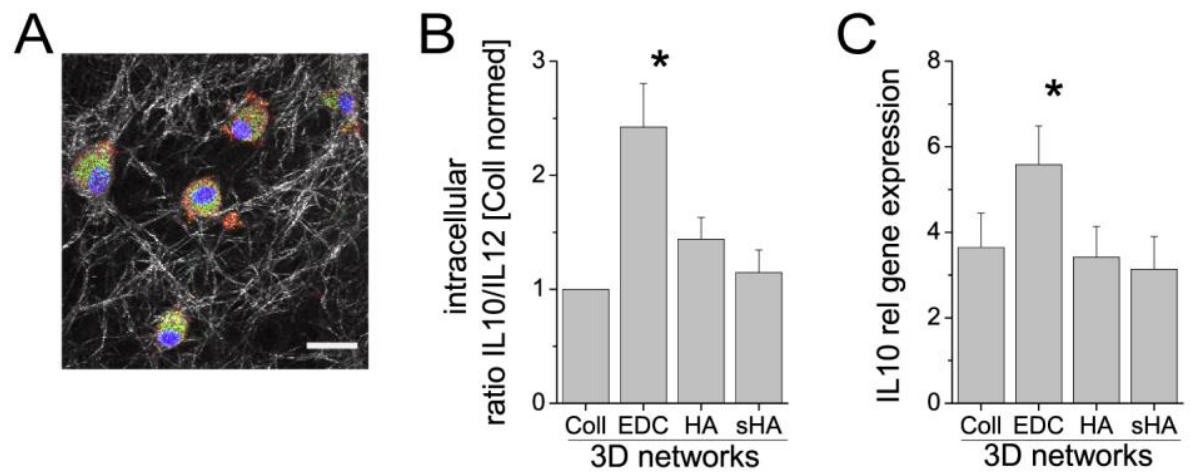

Figure 4. A) Representative confocal laser scanning microscopy images of immunofluorescence analysis of intracellular IL10 (green) and IL12 (red) levels inside the 3D networks. The image was taken in a pure Coll network. Cell nuclei were stained with DAPI (blue). The fibrillar Coll network was visualized in reflection mode (white). Scale bar: $20 \mu \mathrm{m}$. (Separate color images of the multi-channel image are given in Supplemental Figure S3.) B) Ratio of mean fluorescence intensity of intracellular IL10 and IL12 from the immunofluorescence analysis for the different 3D matrices. (at least 20 cells per network from 5 different experiments). C) Gene expression analysis of IL10 by QPCR normalized to reference genes (GNB2L, MRTO4) and relative to GM-MPh without LPS stimulation. $n=7$. *) At the significance level of $p<0.05$, IL10 gene expression and intracellular IL10/IL12 ratio on EDC are significantly higher compared to the values revealed on other networks (Wilcoxon signed-rank test) 
distinguished at this moment. Thus, it is known, that interaction of IL10 with sulfated GAGs can regulate its activity. $^{[47]}$

Our study shows a pronounced effect of increased 3D matrix stiffness (EDC crosslinking) on instructing an anti-inflammatory MPh phenotype despite the presence of the pro-inflammatory GMCSF stimulus. It was also found that this effect is inhibited by GAG integration into the matrix. This important influence of 3D matrix stiffness and the contrasting findings herein and in earlier work ${ }^{[21]}$ for 2D Coll matrices regarding the impact of GAGs emphasize the necessity to apply defined 3D matrices for studies on MPh polarization. Furthermore, it calls for the need to observe various ECM derived stimuli being it dimensionality, matrix mechanics as well as composition (e.g. GAG presence) in such studies. In this context we would like to mention an interesting report, showing an inflammatory MPh response in an EDC crosslinking porcine-derived small intestinal submucosa tissue graft in a rat model. ${ }^{[48]}$ This finding may be differently explained considering our results, as not only the simple stiffness increase by EDC crosslinking might have influenced MPh polarization but also the additional presence of GAGs within these decellularized matrices. The latter might modulate the anti-inflammatory effect of stiffness increase into an inflammatory response like in our experiments. At the current stage we cannot reveal the underlying mechanisms for the differences in MPh polarization by the ECM microenvironment. Whether the antiinflammatory trigger of matrix stiffness might be overruled through interfering effects of cytokine binding or ligand engagement mediated by $\mathrm{HA}$ and sHA needs to be examined in future studies.

However, our study allows to conclude that although network stiffness and 3D context instructs MPh polarization, simple mechanosignaling does not comprehensively explain the underlying cell behavior. Furthermore, a direct influence of EDC chemistry on MPh polarization can also be excluded, too, as EDC was similar presented in GAG functionalized networks and 2D Coll samples.

\section{Conclusion}

The scope of the presented study was to investigate primary human MPh polarization in defined 3D fibrillar matrices with options for modulating stiffness and the presence of sulfated and non-sulfated GAGs. By that, we aimed to mimic the dynamic changes of the ECM microenvironment during wound healing and to reveal their impact on the highly plastic state of MPh phenotypes. Using engineered 3D Coll based matrices with in vivo-like fibrillar characteristics of the network topology, in part similar to dermis and connective tissue, the variation of matrix stiffness by
EDC crosslinking and covalent GAG modification revealed a strong impact on the polarization profile of MPhs. An increase in matrix stiffness by EDC crosslinking led to an instruction of an antiinflammatory MPh phenotype under otherwise inflammatory conditions (GM-CSF differentiation). While the underlying signaling mechanisms remain elusive, our study emphasizes the usage of rationally designed 3D matrices for in vitro studies on wound healing, instead of more artificial 2D settings.

\section{Acknowledgement}

The authors acknowledge the support of grants from EFRE and Free State of Saxony (SAB, grants: 100144684, 100146227), from Deutsche Forschungsgemeinschaft (DFG, grants: SFBTRR67/B3,B10,Z3 and INST 268/293-1 FUGG). This work was supported by the Core Unit FluorescenceTechnologies of the Faculty of Medicine of the University of Leipzig. Stimulating discussions with Colin Logie (Radboud Institute for Molecular Life Sciences) and Ronald Weiss (Institute for Clinical Immunology, Universität Leipzig) are gratefully acknowledged.

\section{References}

[1] G. Charras, E. Sahai, Nat Rev Mol Cell Biol. 2014, 15, 813.

[2] R. O. Hynes, Science. 2009, 326, 1216.

[3] C. Frantz, K. M. Stewart, V. M. Weaver, J Cell Sci. 2010, 123, 4195.

[4] R. O. Hynes, Nat Rev Mol Cell Biol. 2014, 15, 761.

[5] M. Prewitz, F. P. Seib, T. Pompe, C. Werner, Macromol Rapid Commun. 2012, 33, 1420.

[6] L. G. Griffith, M. A. Swartz, Nat Rev Mol Cell Biol. 2006, 7, 211.

[7] J. A. Green, K. M. Yamada, Adv Drug Deliv Rev. 2007, 59, 1293.

[8] E. Cukierman, R. Pankov, D. R. Stevens, K. M. Yamada, Science. 2001, 294, 1708.

[9] M. W. Tibbitt, K. S. Anseth, Biotechnol Bioeng. 2009, 103, 655.

[10] D. R. Coombe, Immunol Cell Biol. 2008, 86, 598.

[11] R. E. Hileman, J. R. Fromm, J. M. Weiler, R. J. Linhardt, Bioessays. 1998, 20, 156.

[12] L. Sorokin, Nat Rev Immunol. 2010, 10, 712.

[13] M. B. Witte, A. Barbul, American J Surgery. 2002, 183, 406.

[14] S. A. Eming, T. Krieg, J. M. Davidson, J Invest Dermatol. 2007, 127, 514.

[15] A. Mantovani, S. K. Biswas, M. R. Galdiero, A. Sica, M. Locati, J Pathol. 2013, 229, 176.

[16] M. G. Rohani, R. S. McMahan, M. V. Razumova, A. L. Hertz, M. Cieslewicz, S. H. Pun, M. Regnier, Y. Wang, T. P. Birkland, W. C. Parks, J Invest Dermatol. 2015, 135, 2377.

[17] S. Franz, S. Rammelt, D. Scharnweber, J. C. Simon, Biomaterials. 2011, 32, 6692.

[18] B. N. Brown, B. D. Ratner, S. B. Goodman, S. Amar, S. F. Badylak, Biomaterials. 2012, 33, 3792.

[19] F. O. Martinez, S. Gordon, F1000Prime Rep. 2014, 6, 13.

[20] D. M. Mosser, J. P. Edwards, Nat Rev Immunol. 2008, 8, 958.

[21] S. Franz, F. Allenstein, J. Kajahn, I. Forstreuter, V. Hintze, S. Moller, J. C. Simon, Acta Biomater. 2013, 9, 5621. 
[22] A. K. Blakney, M. D. Swartzlander, S. J. Bryant, J Biomed Mater Res A. 2012, 100, 1375.

[23] M. Bartneck, K. H. Heffels, Y. Pan, M. Bovi, G. ZwadloKlarwasser, J. Groll, Biomaterials. 2012, 33, 4136.

[24] B. Chevallay, D. Herbage, Med Biol Eng Comput. 2000, 38, 211.

[25] Y. C. Choi, J. S. Choi, B. S. Kim, J. D. Kim, H. I. Yoon, Y. W. Cho, Tissue Eng Part C Methods. 2012, 18, 866.

[26] M. P. Lutolf, J. A. Hubbell, Nat Biotechnol. 2005, 23, 47.

[27] M. V. Tsurkan, K. Chwalek, K. R. Levental, U. Freudenberg, C. Werner, Macromol Rapid Commun. 2010, 31, 1529.

[28] K. Franke, J. Sapudom, L. Kalbitzer, U. Anderegg, T. Pompe, Acta Biomater. 2014, 10, 2693.

[29] L. Kalbitzer, K. Franke, S. Moller, M. Schnabelrauch, T. Pompe, J Mater Chemistry B. 2015, 3, 8902.

[30] J. Zidek, L. Vojtova, A. M. Abdel-Mohsen, J. Chmelik, T. Zikmund, J. Brtnikova, R. Jakubicek, L. Zubal, J. Jan, J. Kaiser, J Mater Sci Mater Med. 2016, 27, 110.

[31] C. B. Raub, V. Suresh, T. Krasieva, J. Lyubovitsky, J. D. Mih, A. J. Putnam, B. J. Tromberg, S. C. George, Biophys J. 2007, 92, 2212.

[32] J. R. Harris, A. Soliakov, R. J. Lewis, Micron. 2013, 49, 60.

[33] S. Leikin, D. C. Rau, V. A. Parsegian, Nat Struct Biol. 1995, 2, 205.

[34] J. M. Lee, H. H. L. Edwards, C. A. Pereira, S. I. Samii, J Mater Sci Mater Med. 1996, 7, 531.

[35] Y. J. Hwang, J. Granelli, J. Lyubovitsky, ACS App/ Mater Interfaces. 2012, 4, 261.

[36] M. Ansorge, N. Rastig, R. Steinborn, T. König, L. Baumann, S. Möller, M. Schnabelrauch, M. Cross, C. Werner, A. G. Beck-Sickinger, T. Pompe, J Control Release. 2016, 224, 59.

[37] V. Hintze, S. Moeller, M. Schnabelrauch, S. Bierbaum, M. Viola, H. Worch, D. Scharnweber, Biomacromolecules. 2009, 10, 3290.
[38] J. Sapudom, S. Rubner, S. Martin, T. Kurth, S. Riedel, C. T. Mierke, T. Pompe, Biomaterials. 2015, 52, 367.

[39] T. Pompe, S. Zschoche, N. Herold, K. Salchert, M. F. Gouzy, C. Sperling, C. Werner, Biomacromolecules. 2003, 4, 1072.

[40] J. L. Hutter, J. Bechhoefer, Review of Scientific Instruments. 1993, 64, 1868.

[41] P. J. Murray, J. E. Allen, S. K. Biswas, E. A. Fisher, D. W. Gilroy, S. Goerdt, S. Gordon, J. A. Hamilton, L. B. Ivashkiv, T. Lawrence, M. Locati, A. Mantovani, F. O. Martinez, J. L. Mege, D. M. Mosser, G. Natoli, J. P. Saeij, J. L. Schultze, K. A. Shirey, A. Sica, J. Suttles, I. Udalova, J. A. van Ginderachter, S. N. Vogel, T. A. Wynn, Immunity. 2014, 41, 14.

[42] K. Wolf, S. Alexander, V. Schacht, L. M. Coussens, U. H. von Andrian, J. van Rheenen, E. Deryugina, P. Friedl, Semin Cell Dev Biol. 2009, 20, 931.

[43] F. A. Verreck, T. de Boer, D. M. Langenberg, M. A. Hoeve, M. Kramer, E. Vaisberg, R. Kastelein, A. Kolk, R. de WaalMalefyt, T. H. Ottenhoff, Proceedings of the National Academy of Sciences of the United States of America. 2004, 101, 4560.

[44] C. S. Chen, M. Mrksich, S. Huang, G. M. Whitesides, D. E. Ingber, Science. 1997, 276, 1425.

[45] S. Prokoph, E. Chavakis, K. R. Levental, A. Zieris, U. Freudenberg, S. Dimmeler, C. Werner, Biomaterials. 2012, 33, 4792.

[46] E. Hsu, T. Osslund, R. Nybo, B. L. Chen, W. C. Kenney, C. F. Morris, T. Arakawa, L. O. Narhi, Protein Eng Des Sel. 2006, 19, 147.

[47] S. Salek-Ardakani, J. R. Arrand, D. Shaw, M. Mackett, Blood. 2000, 96, 1879

[48] S. F. Badylak, J. E. Valentin, A. K. Ravindra, G. P. McCabe, A. M. Stewart-Akers, Tissue Eng Part A. 2008, 14, 1835. 